\title{
The Importance of an Accurate Differential Diagnosis in Chronic Recurrent Multifocal Osteomyelitis
}

\author{
Achille Marino*1, 2, Teresa Gianii, ${ }^{3,4}$ Laura Capirchio ${ }^{4}$, Massimo Basile ${ }^{5}$, Claudio De Filippi ${ }^{5}$, Gabriele \\ Simonini ${ }^{6}$ and Rolando $\mathrm{Cimaz}^{6}$ \\ ${ }^{1} \mathrm{PhD}$ student, University of Florence, Florence, Italy \\ ${ }^{2}$ Department of Pediatrics, Desio Hospital, ASST Monza, Italy \\ ${ }^{3}$ University of Siena, Siena, Italy \\ ${ }^{4}$ Rheumatology Unit, Meyer Children's Hospital Florence, Italy \\ ${ }^{5}$ Radiology Unit, Meyer Children's Hospital, Florence, Italy \\ ${ }^{6}$ Rheumatology Unit, Department of Neurosciences, Psychology, Drug Research and Child Health, Meyer Children's Hospital, University of \\ Florence, Florence, Italy
}

*Corresponding author: Achille Marino, Department of Pediatrics, Desio Hospital, ASST Monza, Italy

\begin{tabular}{|c|c|}
\hline ARTICLE INFO & ABSTRACT \\
\hline $\begin{array}{l}\text { Received: March 01, } 2019 \\
\text { Published: March 15, } 2019\end{array}$ & $\begin{array}{l}\text { Abbreviations: CRMO: Chronic Recurrent Multifocal Osteomyelitis; CRP: C-Reactive Pro- } \\
\text { tein; ESR: Erythrocyte Sedimentation Rate; NSAIDs: Non-Steroidal Anti-Inflammatory }\end{array}$ \\
\hline
\end{tabular}

Citation: Marino A, Giani T, Capirchio L, Basile M, De Filippi C, Simonini G, Cimaz R. The Importance of an Accurate Differential Diagnosis in Chronic Recurrent Multifocal Osteomyelitis. Biomed J Sci \& Tech Res 16(1)-2019. BJSTR. MS.ID.002787.

\section{Background}

AChronic Recurrent Multifocal Osteomyelitis (CRMO) is a rare disease first described by Giedon et al. [1]. It occurs in childhood, typically between 3 and 15 years. Symptoms may be insidious and variable mimicking infections or neoplasms, furthermore laboratory and radiological features are non-specific. Although the exact molecular pathogenesis is unknown, an aberrant regulation of the innate immune system seems to promote the recurrence of bone inflammation [2]. Herein we reported 2 clinical cases with very similar clinical and radiological presentation but different diagnosis and outcome.

\section{Case 1}

A previously healthy 11-year-old boy presented with a 1-month history of low-grade fever and progressive left clavicle pain responsive to non-steroidal anti-inflammatory drugs (NSAIDs). General exam was unremarkable besides tenderness at the central body of left clavicle. Laboratory findings showed normal complete blood count and elevated inflammatory markers [Erythrocyte sedimentation rate (ESR) was $91 \mathrm{~mm} / \mathrm{h}(\mathrm{vn}<30)$ with a C-reactive protein (CRP) measuring $6.1 \mathrm{mg} / \mathrm{dL}(\mathrm{vn}<0.5)]$. Blood and urine cultures resulted negative. Antero-posterior radiographs of left shoulder and clavicle revealed bone osteopenia with a mild periosteal reaction in the central portion of clavicle. Chest X-ray and abdominal ultrasonography resulted negative. Left clavicle MRI was compatible with osteomyeltis (Figure 1a) and an empiric antibiotic therapy was started along with NSAIDs leading to clinical and laboratory improvement. Three months later, after a minor trauma, he started to complain pain at the right arm during the night and daytime along with mild nocturnal fever.

Upon clinical examination, intense pain and restriction of both active and passive movements of right elbow were found. General examination was unremarkable except for a little right 
supra-clavicle lymphadenopathy. At conventional X-ray of the right shoulder and upper limb no changes were documented. Wholebody MRI identified multiple bone lesions located on scapulae, clavicles, upper limbs, vertebrae (D5-11) and sternum (Figure 1b). Bone marrow aspirate resulted negative. An open bone biopsy was than performed and the histology revealed lymphoid cells proliferation. The diagnosis of B-cell lymphoblastic leukemia/ Lymphoblastic lymphoma (B-LBL) was at last confirmed by the histologic examination of the supra-clavicle lymph node.

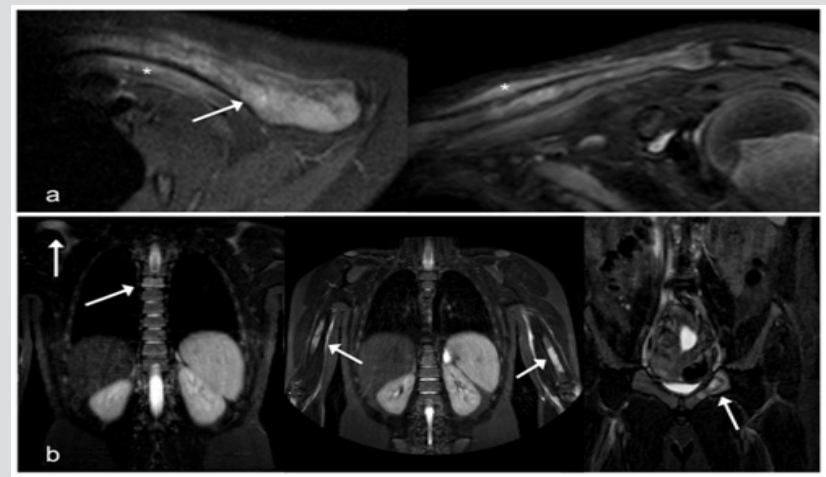

Figure 1:

a) Axial and coronal STIR images of left shoulder MR show diffuse hyperintensity of clavicle (arrow) and abnormal periostal signal intensity extending into adjacent soft tissue (asterisk).

b) Coronal STIR images of Whole-Body MR show multiple hyperintensity bone lesions (right shoulder, bilateral arm , vertebral body D5, and left ischio-pubic ) characterized by edema-like pattern without involving soft tissue.

\section{Case 2}

A 15-year-old boy presented with one-month history of lumbosacral pain. Later on his pain worsened, increasing in intensity and frequency, and appearing during the night rest with inadequate response to NSAIDs.

Laboratory tests revealed slight increase of inflammatory markers (CRP $2.4 \mathrm{mg} / \mathrm{dL}$ and ESR $33 \mathrm{~mm} / \mathrm{h}$ ). Pelvis and lumbosacral X-Ray resulted normal. Whole-body MRI showed multiple bone lesions in the left femur, right sacrum and spine (L4, D12) (Figure 2), confirmed by bone PET. Giving the suspect of a diffuse osteomyelitis, intravenous antibiotic therapy was started. Bone marrow aspirate was performed, excluding the presence of blast cells and extrinsic infiltration. Biopsies from bone lesions were taken and the histological analysis showed a lymphohistiocytic infiltrate. Once neoplasms and infections were excluded, a reconsideration of the available data as the extension, distribution and MRI patterns of the bone lesions suggested the diagnosis of CRMO. Antibiotics were discontinued and a combination therapy with ketorolac, naproxen and tramadol was initiated. Given the good clinical response, naproxen was pursued alone leading to normalization of the inflammatory markers. Whole-body MRI appeared definitively normalized 10 months later and naproxen was than discontinued.
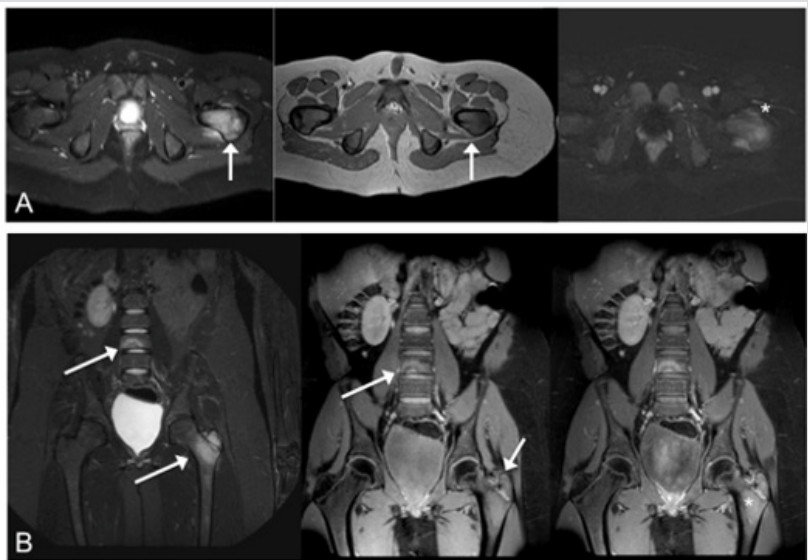

Figure 2:

a) Axial STIR, T1 TSE and contrast-ehanced THRIVE and B)coronal STIR, fat-suppressed T1 and fat-suppressed contrastenhanced T1-weighted images demonstrating abnormal diffuse hyperintensity signal (arrows) and middle enhancement (asterisk) of left femur apophysis and vertebral body (L4) with edema-like pattern. 


\section{Discussion}

CRMO is a rare, often misdiagnosed entity, the impact of which is unclear. The disease onset can be insidious, with non-specific, systemic symptoms as low-grade fever, pain, slight malaise and fatigue. The disease course is characterized by recurrent episodic attacks that may last few weeks to months with a self-limited trend [3].

Bone lesions are often multifocal, most frequently located within the long bones (typically femur and tibia) with symmetrical distribution. However, whole skeleton can be affected. Laboratory investigation may result negative; a mild elevation of white blood cell count and inflammatory markers can also occur [4]. Imaging is very useful to detect bone lesions. Conventional radiography is not able to identify initial lesions, while it can underline the presence of osteolytic lesions with sclerosis (typical of an advanced stage of disease). On the other hand, whole-body MRI (with STIR sequences) is a more sensitivity technique that can detect early bone lesions and unrecognized vertebral compressions [5]. Our two patients are both adolescents' male, in good general condition with a long-term history of localized bone pain disturbing the night rest, uninformative laboratory test, and apparent, similar radiological findings. Histology was diriment for the diagnosis of B-LBL in the first patient and supported the hypothesis of CRMO in the second.

Nevertheless, some clinical and radiological elements might have suggested the correct diagnosis even before bone biopsy. In Case 1 non typical bone site inflammation, presence of right supra-clavicle lymphadenopathy and the lack of improvement on NSAIDS may have suggested another diagnosis Whereas in Case 2 bone lesions detected with MRI had typical location and findings attributable to an autoinflammatory bone disorders and the patient had clinical and radiological improvement with NSAIDs therapy (naproxen). In order not to miss any possible alternative diagnosis, several approaches depending on clinical, radiological and laboratory features have been proposed [4,6]. A careful vigilance and a high index of suspicion are required and mandatory during the early stages. Moreover, a meticulous work-up directed to exclude other possible causes, especially infections and malignancies, is recommended. Although some characteristics of the bone lesions and a careful observation of the radiological features may be suggestive for CRMO, the decision to take a biopsy should be always considered. These two cases point out the difficulties and the caution to correctly diagnose a rare autoinflammatory bone disease.

\section{References}

1. Giedon A, Holthusen W, Masel LF, Vischer D (1972) Subacute and chronic symmetrical osteomyelitis. Ann Radiol (Paris) 15(3): 329-342.

2. Taddio A, Zennaro F, Pastore S, Cimaz R (2017) An update on the pathogenesis and treatment of chronic recurrent multifocal osteomyelitis in children. Paediatr Drugs 19(3): 165-172.

3. Alshammari A, Usmani S, Elgazzar AH, Ashkanani RA (2013) Chronic Recurrent Multifocal Osteomyelitis in Children: A Multidisciplinary Approach is needed to establish a Diagnosis. World J Nucl Med 12(3): 120-123.

4. Taddio A, Ferrara G, Insalaco A, Pardeo M, Gregori M, et al. (2017) Dealing with Chronic Non-Bacterial Osteomyelitis: a practical approach. Pediatr Rheumatol Online J 15(1): 87.

5. Fritz J, Tzaribatchev N, Claussen C, Carrino JA, Horger MS (2009) Chronic recurrent multifocal osteomyelitis: comparison of whole-Body MR imaging with radiography and correlation with clinical and laboratory data. Radiology 252(3): 842-851.

6. Jansson AF, Müller TH, Gliera L, Ankerst DP, Wintergest U, et al. (2009) Clinical score for nonbacterial osteitis in children and adults. Arthritis Rheum 60(4): 1152-1159.

\section{ISSN: 2574-1241}

DOI: 10.26717/BJSTR.2019.16.002787

Achille Marino. Biomed J Sci \& Tech Res

(C) This work is licensed under Creative (c) Commons Attribution 4.0 License

Submission Link: https://biomedres.us/submit-manuscript.php

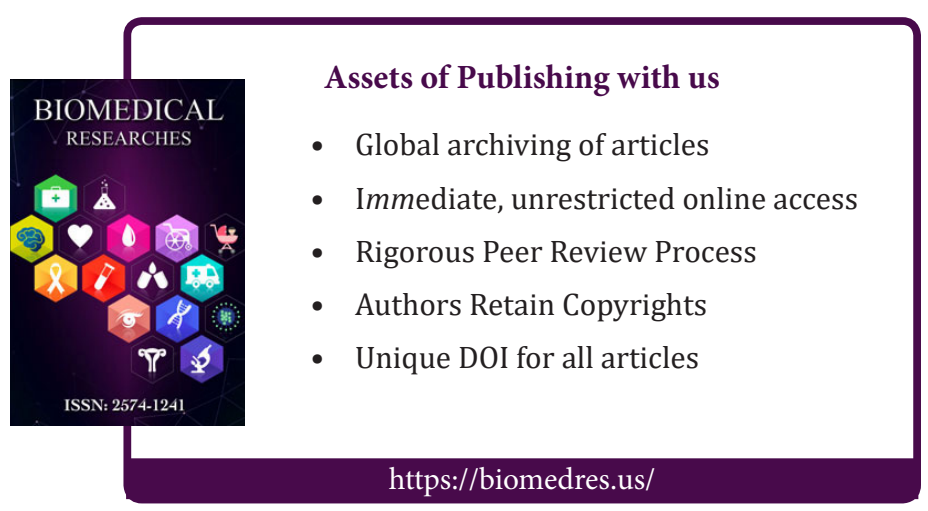

